\title{
Preparation and Properties of Microfibrillated Chitin/Gelatin COMPOSITES
}

\author{
Yifan $\mathrm{Li}^{1}$, Caixin Cao, Ying Pei*, Xueying Liu, Keyong Tang* \\ School of Materials Science and Engineering, Zhengzhou University \\ Zhengzhou 450001, P. R. China. \\ *: Corresponding author E-mail: peiying@zzu.edu.cn,kytangzzu@126.com
}

\begin{abstract}
Microfibrillated chitin/gelatin composite films were prepared by solvent casting method, and the nano-sized microfibrillated chitin as reinforce phase to improve oxygen resistance, water-resistant and mechanical performance in this system. The morphologies were analyzed by scanning electron microscope (SEM), and the mechanical properties were investigated by texture analyzer. Oxygen permeability property, optical property and swelling property were investigated. The results indicated that the elastic modulus and tensile strength of microfibrillated chitin/gelatin composite film reached $2.2 \mathrm{GPa}$ and $74.5 \mathrm{MPa}$ respectively when the content of microfibrillated chitin is 8 wt.\%. The swelling ratio decreased to 11.63 with the 6 wt.\% content of microfibrillated chitin. In addition, chitin microfibrils effectively enhanced the oxygen resistance of composite film without obvious loss of transmittance. This work expects to provide a pathway to improve gelatin performance.
\end{abstract}

\section{Introduction}

With sustainable development and increasing popularities of low carbon economy, the utilization of biomass from nature resources has aroused public attention ${ }^{[1-2]}$. The usage of disposable plastic packings has aroused series of tricky environmental pollution problems. It is urgent to replace synthetic plastic by using biomass materials in the field of food packaging ${ }^{[3-4]}$. Biomass such as proteins and polysaccharides which have been chosen by scientists to applied widely in biomedical or food packaging fields due to their biodegradability, sustainability and renewability ${ }^{[5]}$. In particular, gelatin is a protein obtained from the partial denaturation of native collagen that existed in the bones, tendon and skin of animals, and can be obtained by a controlled hydrolysis reaction [6-8]. Like most proteins, gelatin has good film-forming properties, excellent biocompatibility and biodegradability. Moreover, it features barrier properties against oxygen and aromas at low and intermediate relative humidity ${ }^{[9-10]}$. In previous researches, soy protein isolate/gelatin composites were prepared by compression molding with being able to replace those environmentally unfriendly additives required in making packaging ${ }^{[11]}$. Citric acid-incorporated fish gelatin/chitosan composite films were prepared as active food packaging with good UV barrier properties, showing the excellent ability to reduce the $\mathrm{E}$. coli growth ${ }^{[12]}$.

However, there are some disadvantages of gelatin, which limited its applications. The weak interactions between gelatin molecules make its high brittleness ${ }^{[13]}$. Molecules of hydrophilic nature induce that gelatin is sensitive to water or humid environment, resulting in non-ideal swelling property ${ }^{[14]}$. Numerous efforts have been made to improve the mechanical properties or swelling properties of gelatin by various methods such as chemical crosslinking ${ }^{[15-16]}$, plasticizer and reinforcing modification ${ }^{[17]}$. Chemical agents including glutaraldehyde, formaldehyde would bring healthy and security problems. Although plasticizers could greatly improve gelatin physical performance, they could easily migrate from gelatin matrix when the environment changes, which would have great influence on the modification effect.

Chitin, the most abundant natural polymer besides cellulose on the earth, is a kind of polysaccharide mostly from the shells of crustaceans, insects and other invertebrates ${ }^{[18-19]}$. Chitin features excellent 
biodegradability, antibacterial activity, biocompatibility and low immunogenicity. It's worth noting that microfibrillated chitin could be obtained by high-pressure homogenization, grinding and ultrasonic treatment to be a nano-size biomass material with high surface area and high aspect ratio. Furthermore, microfibrillated chitin possesses ability to form a dense network by inter- and intra-fibril hydrogen bonds. This network structure composed of nano-microfibers benefits the reinforcement of gelatin matrix because of its high strength and stiffness ${ }^{[20-21]}$. In this work, microfibrillated chitin was employed as reinforce phase to improve the performance of gelatin matrix. The structure and properties of composite films were investigated by SEM, texture analyzer, oxygen transmissivity tester and ultraviolet-visible spectrophotometer, expecting to provide a pathway to modify gelatin.

\section{Materials and Methods}

\subsection{Materials}

Gelatin derived from bovine skin (Type B, bloom 250, $p$ I 5-5.5) was purchased from Shanghai Macklin Biochemical Co., Ltd. Chitin powder was supplied by Zhejiang Golden Shell Pharmaceutical Co., Ltd. All other reagents used were of analytical grade and used without further treatment.

\subsection{Chitin Purification}

The purpose of purification is to remove carbonate and protein in carb shells thoroughly, and the procedures were according to the method reported by Cai (2013) ${ }^{[22]}$. Firstly, chitin powder was treated by $5 \%$ sodium hydroxide solution for $6 \mathrm{~h}$ at room temperature, and then washed with deionized water to neutral. Subsequently, the powder was immersed into $7 \%$ hydrochloric acid aqueous solution for 1 day at room temperature. After rinsed with distilled water, the resulting powder was treated with $5 \%$ sodium hydroxide aqueous solution for 2 days. $1.7 \%$ sodium hypochlorite, $0.3 \mathrm{~mol} / \mathrm{L}$ sodium acetate solution treated the above chitin powder for $6 \mathrm{~h}$ at $80{ }^{\circ} \mathrm{C}$. The as-purified chitin powder was rinsed with distilled water and dried for the next use.

\subsection{Microfibrillated Chitin (MFCh) Preparation}

The purified chitin was dispersed into $10 \%$ sodium hydroxide solution under stirring for $2 \mathrm{~h}$ at $60^{\circ} \mathrm{C}$ to a total swelling state. Chitin suspension was homogenized at 1000 bar for 20 times using a highpressure homogenizer equipped with a cooling system (APV 2000, SPX Flow technology Rosista $\mathrm{GmbH}$, German) until obtaining a white milky suspension. The solid content of microfibrillated chitin suspension was determined by dried and weighted.

\subsection{Preparation of MFCh/Gel Composite}

Gelatin was dissolved into distilled water for $2 \mathrm{~h}$ at $50^{\circ} \mathrm{C}$ to obtain gelatin solution. Microfibrillated chitin suspension was mixed with the gelatin solution under agitation for $1 \mathrm{~h}$. Then the mixture was poured into the polytetrafluoroethylene molds and dried at ambient condition (RH 50\%) for 2 days. The composite samples were noted as MFCh/Gel. According to the amount of microfibrillated chitin in gelatin solution: 2, 4, 6, 8 and $10 \mathrm{wt} . \%$, the samples were labeled as MFCh2/Gel, MFCh4/Gel, MFCh6/Gel, MFCh8/Gel, and MFCh10/Gel, respectively. Gelatin film (Gel) without chitin was prepared in the same method. All the film samples were conditioned at $25{ }^{\circ} \mathrm{C}$ and $70 \% \mathrm{RH}$ in a climatic chamber before testing. The average thickness of films was $0.17 \pm 0.027 \mathrm{~mm}$. 


\subsection{Characterization}

The surface and cross section of samples were spilled with gold and observed through a scanning electron microscopy (SEM, Quanta 250, USA) at the accelerating voltage of $20 \mathrm{kV}$ and $5 \mathrm{kV}$. Mechanical properties of the samples were tested with a Texture Analyzer (TAHD-PLUS) at a crosshead speed of $50 \mathrm{~mm} / \mathrm{min}$ using a load cell of $750 \mathrm{~N}$. Tensile modulus $\left(E_{t}\right)$, tensile strength $\left(\sigma_{t}\right)$ and elongation at break $\left(\varepsilon_{\text {break }}\right)$ of samples were determined from stress-strain curves. The resulting values were the average values for every five samples. Swelling experiments were performed by immersing dry films in deionized water until swelling equilibrium. The initial samples were dried at $25^{\circ} \mathrm{C}$ and weighted accurately, noted as $W_{0}$. The weights of swelling samples were noted as $W_{e}$. The swelling capacity $M_{e}$ of the composite was calculated as following:

$$
M_{e}(\%)=\frac{W_{e}-W_{0}}{W_{0}} \times 100
$$

Oxygen Transmission Rate (OTR) measurements have been carried out using an Oxygen Permeability Tester (OX2/231, PERME, China) at $23{ }^{\circ} \mathrm{C}$ abiding by a standard of ASTM D 3985 . The films were installed in a cell where $100 \% \mathrm{O}_{2}$ was flushed on the top side and $100 \% \mathrm{~N}_{2}$ on the bottom side. The amount of $\mathrm{O}_{2}$ transferred through the films was assessed by an oxygen sensor in the $\mathrm{N}_{2}$ gas flow. Ultraviolet-visible spectrophotometer (TU-1950, PERSEE, China) was employed to characterize the optical transmittance of composite films in the wavelength range from 400 to $800 \mathrm{~nm}$.

\section{Results and Discussion}

\subsection{Morphology}

Figure 1 shows the morphology of the chitin powder and the microfibrillated chitin. The morphology of chitin powder is irregular particles. It shows that the high-pressure homogenization achieved effective fiber fibrillation, and the aggregation structure of chitin was separated into smaller fibrils with large surface area (Figure 1a-b). It is observed that the chitin nanofibers display a network structure with the average fibers diameter of around $100 \mathrm{~nm}$, which is distinguished from the morphology of chitin powder (Figure 1c). Effective improvement of matrix in mechanical property could be obtained by the addition of microfibers with higher aspect ratio and network structure ${ }^{[23-24]}$. Therefore, chitin microfibrils could have been a promising reinforce agent for gelatin matrix. The morphologies of MFCh/Gel composites are showed in Figure 2. It is indicated that chitin microfibrils can be dispersed uniformly in the system, showing a very good interfacial adhesion. However, chitin microfibrils aggregated at high concentration, which is in agreement with the following mechanical results.

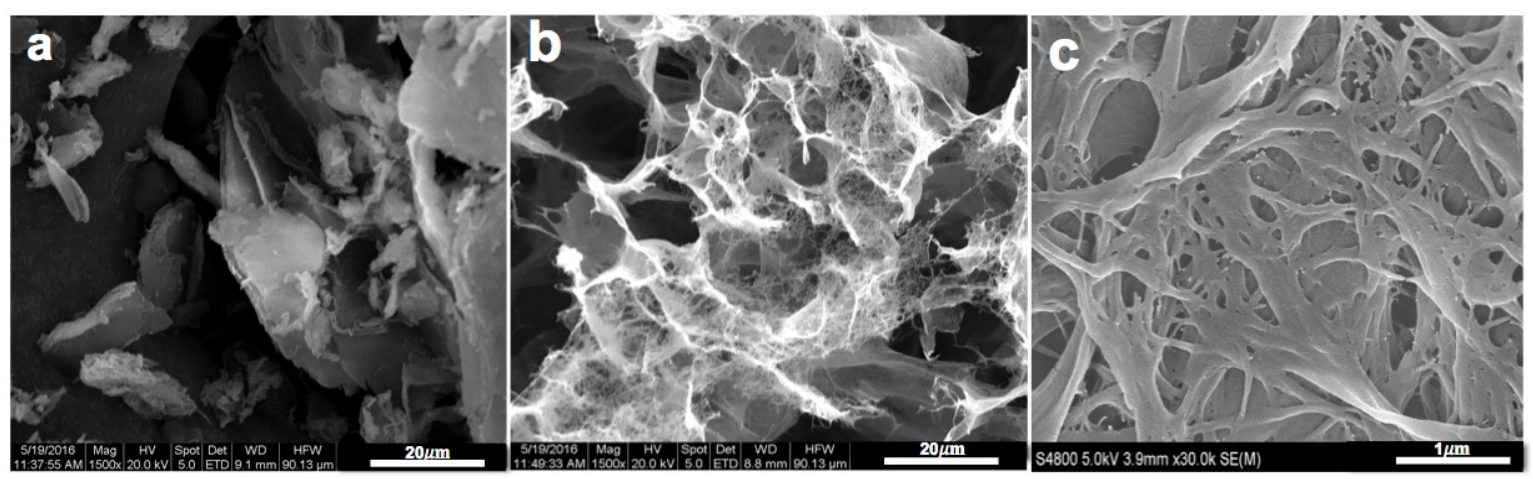

Fig. 1. SEM images of chitin microfibrils. (a) Chitin power, (b-c) microfibrillated chitin treated by highpressure homogenization. 

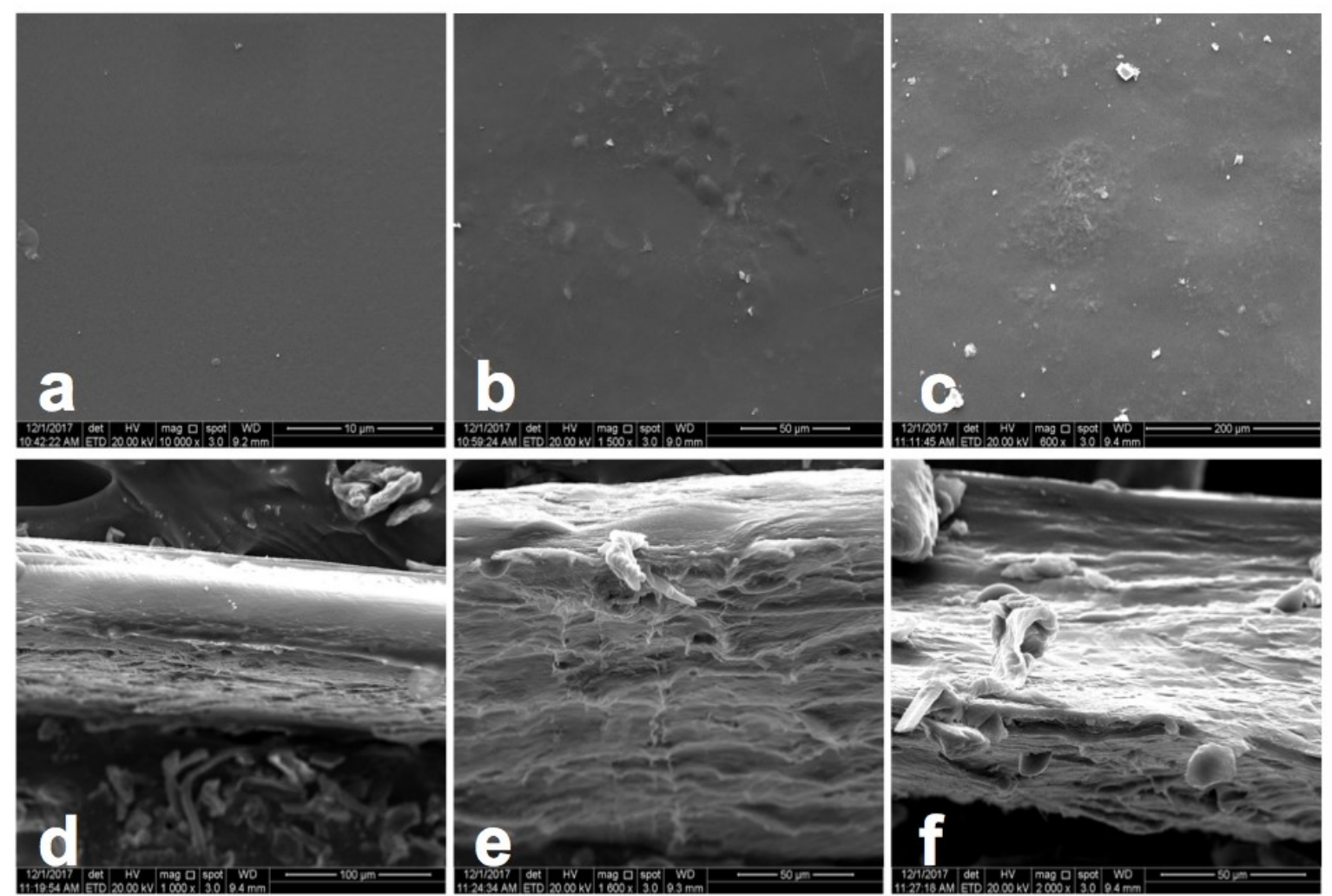

Fig. 2. SEM images of surface (a-c) and cross-sectional (d-f) morphology of MFCh/Gel composites. (a, d) Gel, (b, e) MFCh6/Gel, (c, f) MFCh8/Gel.

\subsection{Tensile Properties of MFCh/Gel Composites}

Figure 3a-c exhibits the effect of the amount of microfibrillated chitin on the tensile properties of the gelatin. The elastic modulus and tensile strength of gelatin are about $1.7 \mathrm{GPa}$ and $61.3 \mathrm{MPa}$, respectively. It is shown that the tensile properties increase with the increase of chitin microfibrils content. When $8 \mathrm{wt} . \%$ microdibrils are incorporated, the optimal mechanical property $\left(E_{t}, \sigma_{t}\right)$ is obtained. The tensile modulus reaches $2.2 \mathrm{GPa}$, and the tensile strength of $\mathrm{CMFh} / \mathrm{Gel}$ is increased from $61.3 \mathrm{MPa}$ to $74.5 \mathrm{MPa}$, an increase of $21.5 \%$, suggesting a good improvement. Due to the high degree microfibrillation, chitin has large aspect ratio and specific surface area with a great quantity of polar hydroxyl groups on the fiber surface, which can have interactions with the amino groups and carboxyl groups of gelatin molecules. The chitin microfibrils not only can bear the load effectively but also prevent the crackle from propagating. When chitin content above $8 \mathrm{wt} . \%$, the tensile strength of the composite films decreases gradually, properly resulting from the aggregations of chitin microfibrils at high concentration. More fibers cannot be dispersed uniformly in the system, and the agglomeration of microfibrillated chitin in the composite film may form stress concentration point when the material is loaded. Furthermore, the elongation at break increased by $26.9 \%$ with the addition of 6 wt.\% micrifibrillated chitin, the maximum value of $\varepsilon_{\text {break }}$ was $7 \%$. It is because some amounts of microfibrillated chitin increase the evenly distribution of stress and decrease the stress concentration. 


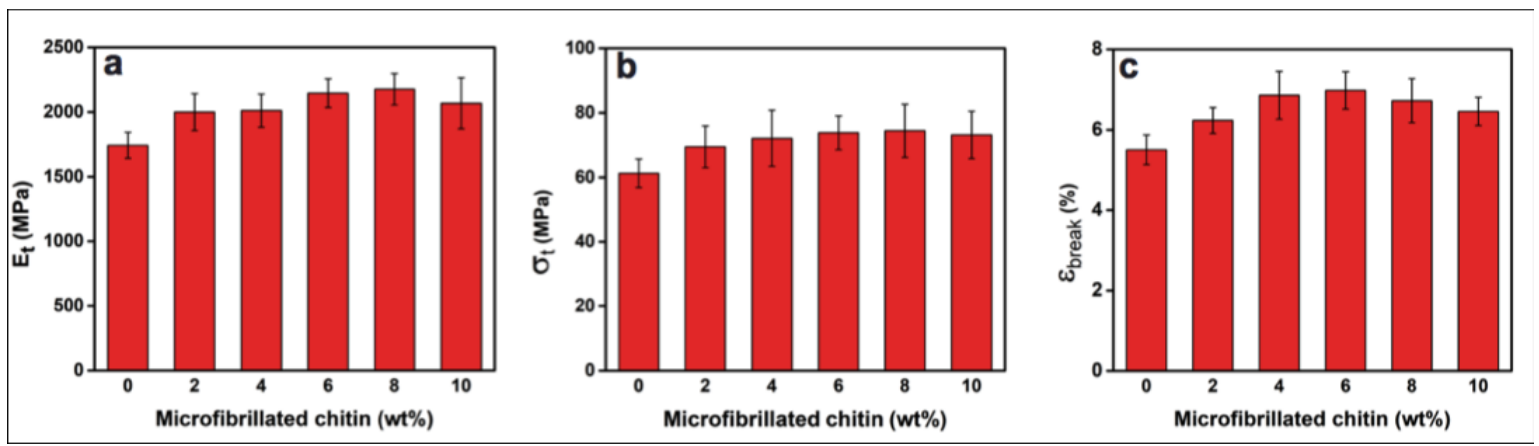

Fig. 3. Tensile properties of gelatin and MFCh/Gel (a) modulus $\left(E_{t}\right)$, (b) strength $\left(\sigma_{t}\right)$ and (c) elongation at break (Ebreak).

\subsection{Swelling Property}

Gelatin has strong hydrophilicity due to the abundant hydrophilic groups such as hydroxyl, carboxyl and amino groups in molecules. It tends to swell or even dissolve when applied in water or humid environment. The swelling ability of the composites influences the physical properties such as shape and mechanical properties. Therefore, the investigation of the swelling ability is very necessary for gelatin system. Swelling ratios of gelatin and MFCh/Gel composites are shown in Figure 4 . It is observed that with increasing chitin content, the swelling ratios of the composite films decrease at first and then increase. When the content of microfibrillated chitin is $6 \mathrm{wt} . \%$, a minimum swelling ratio of 11.63 is obtained, which is $14.43 \%$ lower than that of gelatin film. Therefore, the addition of chitin microfibrils can decrease the swelling ratio and the water sensitivity of gelatin matrix. On one hand, the interactions between gelatin and chitin microfibrils replace some interactions between gelatin and water molecules, reducing the hydrophilic tendency of gelatin system. On the other hand, the network structure of microfibrillated chitin could effectively depress the water uptake through capillary action in gelatin matrix, resulting in the improvement of swelling property ${ }^{[25]}$. However, with the increase of microfibrillated chitin content, the swelling ratio of composite increases on the account of severe phase separation between chitin fiber and gelatin.

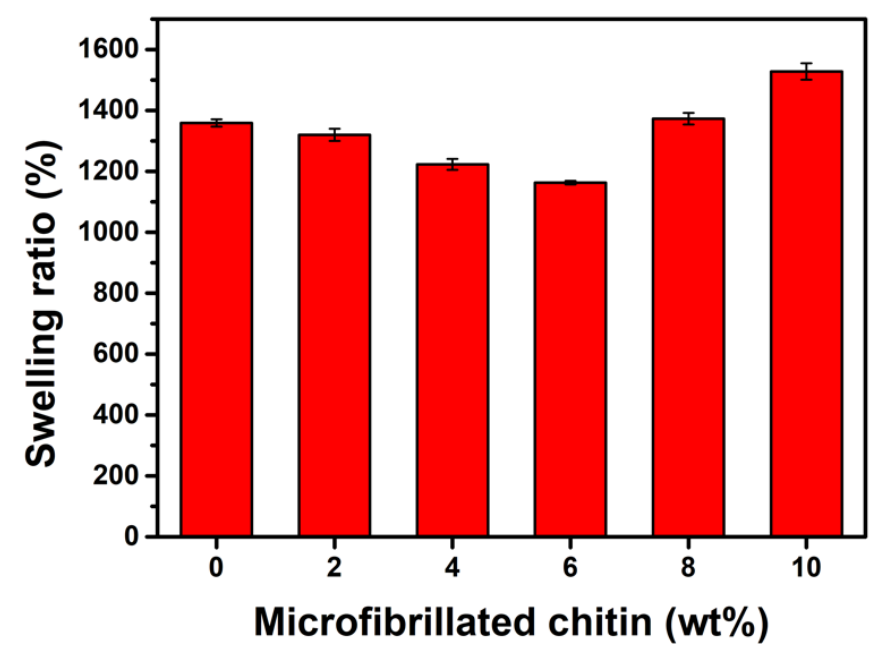

Fig. 4. Swelling property of gelatin and MFCh/Gel.

\subsection{Oxygen Permeability Property}

Gas barrier performance of packaging materials is a key parameter because good oxygen resistance can effectively help slow food deterioration and extend shelf life. Oxygen transmission rate (OTR) 
of gelatin and composites is presented in Figure 5 . The addition of chitin microfibrils significantly decreases OTR compared with that of the gelatin film especially for $10 \mathrm{wt} . \%$ microfibrillated chitin content. Microfibrillated chitin with network structure can create a longer diffusion path for oxygen permeation, resulting in the decreasing in the permeability of composites ${ }^{[26]}$.

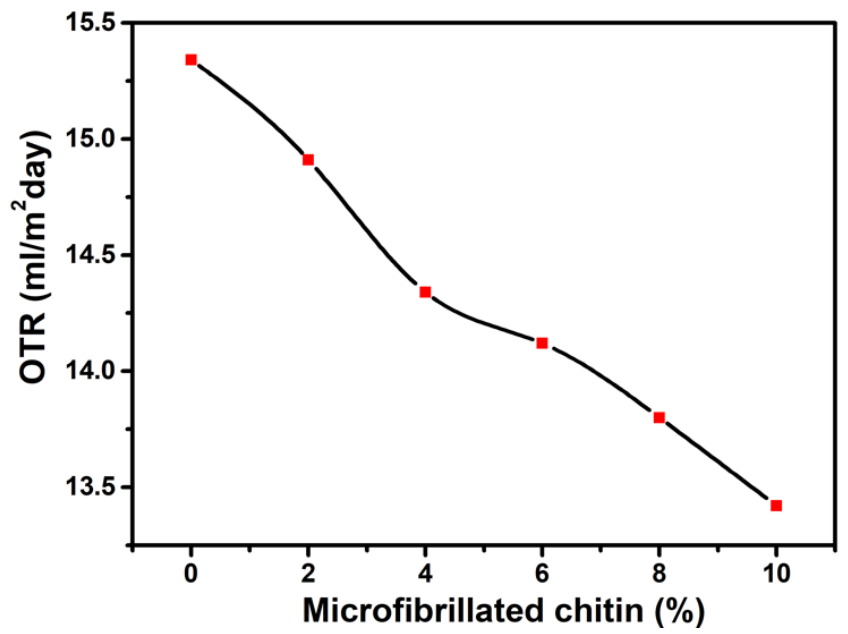

Fig. 5. Oxygen Transmission Rate (OTR) of gelatin and MFCh/Gel.

\subsection{Optical Property}

The optical property is of great importance for packaging materials. Light transmission of the $\mathrm{MFCh} / \mathrm{Gel}$ films is shown in Figure 6 . It indicates that the gelatin film has a high transmittance and can reach about $90 \%$ in the visible wavelength range from 400 to $800 \mathrm{~nm}$. An obvious UV resistivity for gelatin and MFCh/Gel films appears in the wavelength range of 200-250 nm due to the ability of UV radiation absorbance for protein material. The films remain relatively high transparency with low content of microfibrillated chitin (less than 8 wt.\%) due to microfibrillated chitin's homodisperse in the gelatin matrix ${ }^{[27]}$. With increasing the amount of microfibrillated chitin, the light transmittance of the composite film decreases gradually, which may be due to the agglomerations of microfibrillated chitin and phase separations in the composite system. However, the composite film can maintain a good light transmittance of above $60 \%$ when addition of $10 \mathrm{wt} . \%$ microfibrillated chitin content, which could be acceptable in packing applications.

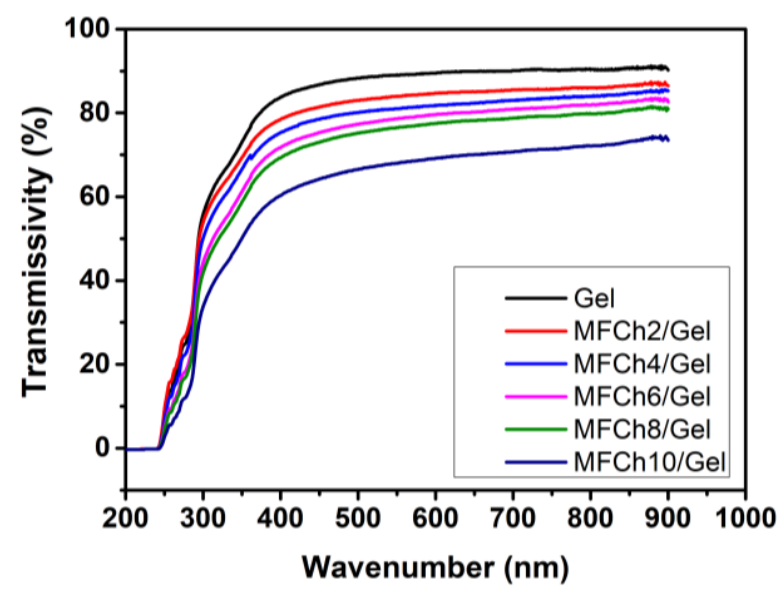

Fig. 6. Effect of microfibrillated chitin content on the optical property of the gelatin and MFCh/Gel. 


\section{Conclusions}

Chitin can be separated into microfibrils successfully by high-pressure homogenization to obtain microfibrillated chitin with nanoscale diameter and network morphology. Owing to network structure and abundant hydroxyl groups, microfibrillated chitin played the role of a reinforcing agent in gelatin matrix to improve the mechanical properties. When the addition of microfibrillated chitin was $8 \mathrm{wt} . \%$, the modulus and tensile strength increased to $2.2 \mathrm{GPa}$ and $74.5 \mathrm{MPa}$, which improved $29.4 \%$ and $21.5 \%$ compared to that of gelatin film. The swelling ability of composite films dramatically decreased with 6 wt.\% chitin microfibrils content, due to the interactions between gelatin and chitin microfibrils and the depression of water uptake by the network structure of microfibrillated chitin through capillary action. The incorporation of chitin microfibrils greatly improved oxygen barrier property of the biomass composites since that chitin microfibrils could create a longer diffusion path for oxygen permeation. Moreover, the composite film exhibited relatively good optical transmittance property in the visible range. This work expects to provide a pathway to improve gelatin performance and a new composite material for packaging applications.

\section{References}

1. Mu C, Guo J, Li X, et al. Preparation and properties of dialdehyde carboxymethyl cellulose crosslinked gelatin edible films, J. Food Hydrocolloids. 27(1) (2012) 22-29.

2. Pena $C, D$ Caba K, Eceiza A, et al. Enhancing water repellence and mechanical properties of gelatin films by tannin addition, J. Bioresour Technol. 101(17) (2010) 6836-6842.

3. Vieira M G A, Silva M A D, Santos $L O D$, et al. Natural-based plasticizers and biopolymer films: A review, J. European Polymer Journal. 47(3) (2011) 254-263.

4. Wang J, Wang J, Zhang K, et al. Preparation and characterization of CST/CS/PVA/GEL blend membrane, J. Materials Review. 35(11) (2012) 2689-2693.

5. Chambi H, Grosso C, Edible films produced with gelatin and casein cross-linked with transglutaminase, J. Food Research International. 39(4) (2006) 458-466.

6. Cai Z, Kim J, Preparation and characterization of novel bacterial cellulose/gelatin scaffold for tissue regeneration using bacterial cellulose hydrogel, J. Journal of Nanotechnology in Engineering and Medicine. 1(2) (2010) 1-6.

7. Hashim D M, Man Y, Norakasha R, et al. Potential use of fourier transform infrared spectroscopy for differentiation of bovine and porcine gelatins, J. Food Chemistry. 118(3) (2010) 856-860.

8. Martucci J F, Ruseckaite $R A$, Biodegradation of three-layer laminate films based on gelatin under indoor soil conditions, J. Polymer Degradation \& Stability. 94(8) (2009) 1307-1313.

9. Lu A, Ma Z, Zhuo J, et al. Layer-by-layer structured gelatin nanofiber membranes with photoinduced antibacterial functions, J. Journal of Applied Polymer Science. 128(2) (2013) 970-975.

10. Martucci J F, Ruseckaite R A, Biodegradation of three-layer laminate films based on gelatin under indoor soil conditions, J. Polymer Degradation \& Stability. 94(8) (2009) 1307-1313.

11. Guerrero $P$, Stefani $P M$, Ruseckaite $R A$, et al. Functional properties of films based on soy protein isolate and gelatin processed by compression molding, J. Journal of Food Engineering. 105(1) (2001) 65-72.

12. Uranga J, Puertas A I, Etxabide A, et al. Citric acid-incorporated fish gelatin/chitosan composite films, J. Food Hydrocolloids. 86(2019) 95-103.

13. George J, Siddaramaiah, High performance edible nanocomposite films containing bacterial cellulose nanocrystals, J. Carbohydrate Polymers. 87(3) (2012) 2031-2037.

14. Karnnet S, Potiyaraj P, Pimpan V, Preparation and properties of biodegradable stearic acid-modified gelatin films, J. Polymer Degradation \& Stability. 90(1) (2005) 106-110.

15. Kim S, Nimni M, Yang Z, et al, Chitosan/gelatin-based films crosslinked by proanthocyanidin, J. Journal of Biomedical Materials Research Part B: Applied Biomaterials. 75B(2) (2005) 442-450.

16. Matsuda S, Se N, Iwata H, et al. Evaluation of the antiadhesion potential of UV cross-linked gelatin films in a rat abdominal model, J. Biomaterials. 23(14) (2002) 2901-2908.

17. Fakhreddin H S, Rezaei M, Zandi M, et al. Preparation and functional properties of fish gelatin-chitosan blend edible films, J. Food Chemistry. 136(3-4) (2013) 1490-1495.

18. Chai $P$, Zhang $W$, Jin X, The new exploiting and researching trends of chitin/chitosan, J. Chemistry. (7) (1999) 8-11. 


\section{Congress of IULTCS}

19. Simpson B K, Gagne N, Simpson M V, Bioprocessing of chitin and chitosan, in: AM Martin (Eds.), Fisheries Processing. London: Chapman and Hall, 1994, pp. 155-173.

20. Liu M , Huang J , Luo B , et al. Tough and highly stretchable polyacrylamide nanocomposite hydrogels with chitin nanocrystals, J. International Journal of Biological Macromolecules. 78 (2015) 23-31.

21. Salaberria A M , Diaz R H, Labidi J, et al. Role of chitin nanocrystals and nanofibers on physical, mechanical and functional properties in thermoplastic starch films, J. Food Hydrocolloids 46 (2015) 93-102.

22. Duan B, Chang C, Ding B, et al. High strength films with gas-barrier fabricated from chitin solution dissolved at low temperature, J. Journal of Materials Chemistry A. 1(5) (2013) 1867-1874.

23. George J, Siddaramaiah, High performance edible nanocomposite films containing bacterial cellulose nanocrystals, J. Carbohydrate Polymers. 87(3) (2012) 2031-2037.

24. Eichhorn S J, Dufresne A, Aranguren M, et al. Review: current international research into cellulose nanofibres and nanocomposites, J. Journal of Materials Science. 45(1) (2010) 1.

25. Zheng X, Liu J, Pei Y, et al. Preparation and properties of sisal microfibril/gelatin biomass composites, J. Composites Part A Applied Science \& Manufacturing. 43(1) (2012) 45-52.

26. Belbekhouche S, Bras J, Siqueira G, et al. Water sorption behavior and gas barrier properties of cellulose whiskers and microfibrils films, J. Carbohydrate Polymers. 83(4) (2011) 1740-1748.

27. Ifuku S, Ikuta A, Egusa M, et al. Preparation of high-strength transparent chitosan film reinforced with surfacedeacetylated chitin nanofibers, J. Carbohydrate Polymers. 98(1) (2013) 1198-1202. 